\title{
THE THERAPEUTIC EFFECT OF PHYSICAL ACTIVITY IN A DAY-HOSPITAL PATIENT WITH ANOREXIA NERVOSA
}

\author{
Kathryn Hughes ${ }^{1}$, David Woodgate ${ }^{1}$, Sarah J. Halford ${ }^{1}$, Mary Cowan $^{1}$ \& Hubertus Himmerich ${ }^{1,2}$ \\ ${ }^{I}$ Bethlem Royal Hospital, South London and Maudsley NHS Foundation Trust, London, UK \\ ${ }^{2}$ Department of Psychological Medicine, King's College London, London, UK
}

received: 30.1.2019;

revised: 14.5.2019;

accepted: 27.5.2019

$* * * * *$

\section{INTRODUCTION}

Many presentations of anorexia nervosa (AN) are characterised by frequent engagement in excessive physical exercise (PE) (Zunker et al. 2011). Therefore, the treatment of AN includes the restriction of PE, and in some severe cases individuals are prescribed bed rest (Moola et al. 2015). Clinical observation has highlighted that advice to completely refrain from physical activity often drives the compulsion to exercise secretly, serving only to reinforce the isolative and shameful experience of an eating disorder (ED). Likewise, clinicians can feel frustrated that advice to refrain from $\mathrm{PE}$ is not taken (Beumont et al. 1994).

The majority of patients with AN experience loss of muscle mass (El Ghoch et al. 2017). For this reason, resistance training seems sensible to prevent muscle atrophy (Fernández-del-Valle et al. 2016). Additionally, PE has been shown to promote bone health (Santos et al. 2017) which is beneficial as AN is typically associated with low bone mass, impaired bone structure, and reduced bone strength; all of which contribute to increased fracture risk (Misra \& Klibanski 2014). Alongside the physical health benefits, PE can help improve mood, quality of life and wellbeing (Moola et al. 2015).

However, the research into PE in the treatment of AN is limited and there are no standardised guidelines (Zunker et al. 2011) despite considerable efforts on an international level (BSOE 2018). During such efforts, e.g. the Ersmus Plus project "Brighter Side of Exercise" (BSOE 2018) it has been repeatedly noticed that fitness trainers are often uncertain about the appropriateness of specific exercises for patients with AN.

\section{CASE}

This case report describes a female patient in her mid-30s who suffers from AN according to the Diagnostic and Statistical Manual of Mental Disorders (DSM-5) (American Psychiatric Association 2013). She first presented with mild eating difficulties during her adolescence but only received help once in her 20s from a dietician and counsellor as part of the Student Health Services. Following this treatment, she was able to manage without ED services for 10 years, but her ED behaviours began to re-develop following a miscarriage and significant stress at work. Before admission, she experienced significant weight loss while eating only 400 calories per day.

Considerable occupational disruption and imbalance was a key feature with daily life being dominated by a pattern of over-exercising and restriction of food. This over-activity was identified as a measure to cope emotionally, with her using activity to create an "artificial schedule" that enabled her to experience a sense of being efficient. As a result, time use and patterns of activity excluded restoration, leisure and social connectedness with others.

\section{MULTI-DISCIPLINARY FOCUSED INTERVENTION}

The patient was referred to the South London and Maudsley (SLaM) ED Service by her general practitioner at a weight of $35 \mathrm{~kg}$ (BMI: $15 \mathrm{~kg} / \mathrm{m} 2$ ). Following this referral, she began with the "Step-Up" programme at Bethlem Royal Hospital (BRH) which uses a client-centred approach to support individuals with EDs to work towards recovery. It offers occupational therapy (OT), nursing, dietetic, psychological and psychiatric support in the form of group work, individual sessions and outreach community work. Patients work collaboratively with staff towards achieving goals that they set to improve their quality of life.

\section{PHYSICAL EXERCISE INTERVENTION}

For this patient, a particular goal was to incorporate healthy exercise into her life and to build confidence in her body. The PE team at BRH created a client-owned programme which involved education on the principles of training and components of fitness. It had an emphasis on resistance training to increase muscle mass. The programme was balanced for antagonistic muscle groups, which involved a mixture of single muscle or muscle group uniplanar exercise, and more compound movements and functional exercise. It was emphasised that exercise is not a chore but should be built around social interaction and should be meaningful. 
Table 1. Key elements of the therapeutic use of physical exercise (PE) in a day-care patient with anorexia nervosa (AN) Integration of $\mathrm{PE}$ into a multimodal treatment concept consisting of:

- Occupational therapy

- Nursing

- Diet counselling

- Psychological therapy

- Psychiatric and medical care

Preparation of PE in AN:

- Collection of information about the patient's history and experience around PE

- Identification of individual goals for PE which are helpful for recovery

- Individualised education on PE

- Instructions on moderate strength training

- Introduction to machines and equipment

- Individualised training plan

Principles for the use of PE in AN:

- Strength training balanced for antagonistic muscle groups

- Resistance training

- Training in body perception

- Promotion of social interactions

- Purpose of PE not related to the ED symptoms

Specific exercises using:

- Cable crossover

- Free weights

- Resistance bands

- Own bodyweight

The patient met with her instructor to discuss exercise history, interests, social support, aims and different forms of exercise and activity. She was shown around the machines and equipment, and a basic programme was devised. Emphasis was put on low-level resistance training using a cable crossover, free weights, resistance bands and bodyweight. She started with training once per week progressing to twice. During the treatment period, the instructor supported her to focus on recovery. The whole intervention lasted for six months. Table 1 summarizes the PE treatment.

\section{OUTCOME}

On discharge from "Step-Up", the patient had made significant gains in weight to $45.5 \mathrm{~kg}$ (BMI: $18 \mathrm{~kg} / \mathrm{m} 2$ ). The ED Examination Questionnaire (EDE-Q) score at discharge was below (Giovazolias et al. 2013) the clinical cut off of 4 .

\section{DISCUSSION}

This case report reveals that significant benefits can be made from using PE in the form of resistance training as a treatment modality within a day care ED service. The individual discussed was able to increase her weight to a healthy BMI and improve in terms of ED psychological symptomatology.

This result is in line with previous research which suggests that strength training is beneficial for patients with AN regarding their physical and mental health (Fernández-del-Valle et al. 2016). In the past, PE has been thought of as contraindicative of weight restoration (Moola et al. 2015). However, the Step-Up team has found this to be an important part of the reported patient's recovery. It should be noted that PE alone would not be sufficient to support weight restoration and the combination of different treatment options available at Step-Up were all key factors in the successes of the individual described.

\section{CONCLUSIONS}

This case report, however, has its limitations as it is difficult to generalise the outcome in one case to a larger population. Therefore, future research should look into the use of PE in the treatment of AN to further our understanding of its role in recovery. Nevertheless, it provides specific information about how we planned and performed PE in a patient with AN and thus makes a concrete suggestion for an intervention which can be evaluated in a larger study sample.

\section{Acknowledgements:}

We thank the patient reported in this article for agreeing to have her case published.

Conflict of interest: None to declare. 


\section{Contribution of individual authors:}

Kathryn Hughes drafted the whole manuscript.

David Woodgate contributed written information about the PE intervention for the patient.

Sarah J. Halford \& Mary Cowan added key features of the Step-Up programme and the patient's history.

Hubertus Himmerich formatted the draft into a scientific letter.

All authors discussed and amended the manuscript and gave their final approval before submission.

\section{References}

1. American Psychiatric Association: Diagnostic and statistical manual of mental disorders. American Psychiatric Publishing, Arlington, 2013

2. Beumont PJ, Arthur B, Russell JD \& Touyz SW: Excessive physical activity in dieting disorder patients: proposals for a supervised exercise program. Int J Eat Disord 1994; 15:21-36

3. BSOE: Fitnes Učilište: Brighter Side of Exercise 2018; Available at: http://bsoe.eu/ [Accessed 20 Dec. 2018]
4. El Ghoch M, Pourhassan M, Milanese C, Müller MJ, Calugi S, Bazzani PV et al.: Changes in lean and skeletal muscle body mass in adult females with anorexia nervosa before and after weight restoration. Clin Nutr 2017; 36:170-178

5. Fernández-del-Valle M, Larumbe-Zabala E, MorandeLavin G \& Perez Ruiz M: Muscle function and body composition profile in adolescents with restrictive anorexia nervosa: does resistance training help? Disabil Rehabil 2016; 38:346-353

6. Giovazolias $T$, Tsaousis I \& Vallianatou C: The factor structure and psychometric properties of the Greek version of the Eating Disorders Examination Questionnaire (EDE-Q). Eur J Psychol Assess 2013

7. Misra $M \&$ Klibanski A: Anorexia nervosa and bone. $J$ Endocrinol 2014; 221:R163-R176

8. Moola FJ, Gairdner S \& Amara C: Speaking on behalf of the body and activity: investigating the activity experiences of Canadian women living with anorexia nervosa. Ment Health Phys Act 2015; 8:44-55

9. Santos L, Elliott-Sale KJ \& Sale C: Exercise and bone health across the lifespan. Biogerontology 2017; 18:931-946

10. Zunker C, Mitchell JE \& Wonderlich SA: Exercise interventions for women with anorexia nervosa: a review of the literature. Int J Eat Disord 2011; 44:579-584

Correspondence:

Kathryn Hughes, PhD, PG certificate in Psychiatric Therapeutics

Step-Up Eating Disorders Service, Tyson West 2, Bethlem Royal Hospital,

Monks Orchard Road, Beckenham, BR3 3BX, UK

E-mail: kathryn.hughes@slam.nhs.uk 